\title{
PROBLEMATIZAÇÃO COMO MÉTODO ATIVO DE ENSINO-APRENDIZAGEM: ESTUDANTES DE FARMÁCIA EM CENÁRIOS DE PRÁTICA
}

\author{
PROBLEMATIZATION AS AN ACTIVE METHOD OF TEACHING AND LEARNING: \\ PHARMACY STUDENTS IN PRACTICE SETTINGS
}

\author{
Rinaldo Henrique Aguilar da Silva ${ }^{1}$ \\ Soraida Sozzi Miguel ${ }^{2}$ \\ Luciana Scapin Teixeira ${ }^{3}$
}

\begin{abstract}
Resumo O objetivo deste trabalho é realizar uma reflexão sobre a implantação de metodologia ativa de ensino-aprendizagem da problematização nos cenários de prática da graduação em Farmácia. A problematização vem sendo utilizada como estratégia de ensino visando à formação de profissionais mais críticos, reflexivos, capazes de trabalhar em equipe e de aprender juntos. O farmacêutico com esse perfil é capaz de atuar no Sistema Único de Saúde (SUS) articulado com o contexto social, contribuindo para a melhoria da qualidade de vida das pessoas, conforme preconizam as Diretrizes Curriculares Nacionais. Para isso, foram analisados qualitativamente os instrumentos de avaliação preenchidos pelos estudantes que destacaram as principais fragilidades e fortalezas do processo. Foi possível analisar os seguintes aspectos: cenários de prática; orientação discente; reorientação da formação e assistência. Conclui-se que, apesar dos grandes desafios ainda existentes, incluindo a inadequada capacitação dos docentes, esse modelo de educação está em consonância com o objetivo proposto.
\end{abstract}

Palavras-chave ensino superior; Farmácia; problematização.
Abstract The purpose of this study was to reflect on the deployment of an active teaching and learning methodology in practical settings in undergraduate Pharmacy studies. Problematization has been used as a teaching strategy aimed at training more critical, reflective professionals capable of working and learning together. A pharmacist with this profile is capable of working in the Single National Health System (SUS) integrated with the social context and contributing to improving people's quality of life, as advocated by the National Curriculum Guidelines. For this, the assessment tools provided by the students were analyzed qualitatively, and the main weaknesses and strengths of the process were highlighted. It was possible to analyze the following aspects: practical settings, student orientation, retraining, and assistance. We conclude that, despite the fact that major challenges still remain, including inadequate teacher training, this educational model is consistent with the proposed goal.

Keywords higher education; Pharmacy; problematization. 


\section{Introdução}

A história do ensino farmacêutico no Brasil (com início datado de 1832) pode ser contada com base nas tentativas de alteração e uniformização curriculares. Mais expressivamente influem sobre a situação atual os currículos mínimos de 1962 (parecer CFE n. ${ }^{\circ}$ 268) e de 1969, que regularam a graduação em farmácia até 2002. Na década de 1980, o papel do farmacêutico na sociedade passou a ser tema de discussão na categoria, acompanhado necessariamente da discussão sobre a educação farmacêutica.

A publicação das Diretrizes Curriculares Nacionais (DCN) de 2002 (resolução CNE/CES n. ${ }^{\circ}$ 02/2002) foi um importante marco histórico para a profissão, ao determinar que a formação do farmacêutico deve ter como foco a preparação para atuar no Sistema Único de Saúde (SUS) articulado ao contexto social com participação e contribuição para o desenvolvimento da sociedade. Após a publicação das DCN, as dificuldades geradas na sua interpretação e aplicação direcionaram as discussões na área da educação farmacêutica, que ficaram concentradas nas formas de implementar esse novo modelo curricular.

Podemos afirmar que o ensino farmacêutico vive uma crise de paradigmas que se caracteriza por uma mudança conceitual, ou uma mudança de visão do mundo, consequência de uma insatisfação com modelos anteriormente predominantes de explicação. A crise de paradigmas leva geralmente a uma mudança, sendo que as mais radicais consistem em revoluções científicas. Aliar educação, e não somente ensino, atualização de conhecimentos, questionamento construtivo e práticas diferenciadas exigem um novo perfil para o professor tanto quanto para o aluno e futuro farmacêutico. Assim, concebe-se um profissional que transcenda a formação técnica e passe a considerar o paciente como um ser integral que merece uma abordagem biopsicossocial.

Um dos campos em que os farmacêuticos devem atuar ativamente nessa perspectiva refere-se à assistência farmacêutica. Existe um razoável consenso hoje no país em adotar a definição expressa no documento da Política Nacional de Medicamentos que define a Assistência Farmacêutica como

Grupo de atividades relacionadas com o medicamento destinadas a apoiar as ações de saúde demandadas por uma comunidade. Envolve o abastecimento de medicamentos em todas e em cada uma de suas etapas constitutivas, a conservação e o controle de qualidade, a segurança e a eficácia terapêutica dos medicamentos, o acompanhamento e a avaliação da utilização, a obtenção e a difusão de informação sobre medicamentos e a educação permanente dos profissionais de saúde, do paciente e da comunidade para assegurar o uso racional de medicamentos (Brasil, 1999). 
“O tratamento medicamentoso é o escolhido para muitas doenças e distúrbios. Foi estimado, entretanto, que a adesão média seja em torno de 50\% (Blenkinsopp et al., 2000; Marinker e Shaw, 2003; OMS, 2003). O termo 'adesão' foi definido como 'a extensão com que o paciente segue as instruções recebidas' (Blenkinsopp et al., 2000, p.10; WHO, 2003)" (Puumalainen e Kansanaho, 2009, p. 7)

Essa abordagem não considera o paciente como sujeito ativo ao lidar com sua própria condição. Ao contrário, nessa abordagem o paciente é considerado como um objeto de decisões tomadas por um profissional de saúde. Na abordagem fundamentada na adesão, o aconselhamento ao paciente é visto como um processo em que um profissional da saúde, autoritariamente, transmite informação a um sujeito passivo. Dessa forma, está fundamentado no conceito da aprendizagem tradicional.

Considerando que a maioria dos estudantes aprende por meio de modelos tradicionais, como esperar que eles, na sua atuação profissional, possam agir de maneira diferente?

Esse paradigma cartesiano, ou clássico, dominou nossa cultura durante centenas de anos, ao longo dos quais modelou a sociedade ocidental. Na saúde em geral, ainda predomina até os dias de hoje. A influência do paradigma cartesiano sobre as ciências da saúde resultou no chamado modelo biomédico que constitui o alicerce conceitual da ciência moderna.

No curso de Farmácia da Faculdade de Ciências Médicas e da Saúde de Juiz de Fora (FCMS/JF), essa mudança de paradigma está alicerçada na vivência do método pedagógico da problematização, segundo Bordenave e Pereira (1991). A escolha dessa estratégia pedagógica tem como objetivo formar profissionais aptos ao exercício da profissão, capazes de continuar a aprender a aprender para toda a vida com visão ética, humanística e responsabilidade social, que são qualidades importantes na construção do SUS.

A problematização é realizada em cinco etapas: observação da realidade - é a realização pelos estudantes da tarefa social e concreta, com base em um tema ou unidade de estudo; pontos-chave - os estudantes são levados a refletir primeiramente sobre as possíveis causas da existência do problema em estudo; teorização - estudo, investigação propriamente dita (os estudantes se organizam tecnicamente para buscar as informações de que necessitam sobre o problema onde quer que elas se encontrem, dentro de cada pontochave já definido); hipóteses de solução - todo o estudo realizado deverá fornecer elementos para os estudantes, crítica e criativamente, elaborarem as possíveis soluções; aplicação à realidade - são discutidas e encaminhadas à equipe de saúde as estratégias de intervenção.

Essas etapas são articuladas entre si e possibilitam que o estudante possa desenvolver a capacidade de aprender a aprender, a aplicar o conhecimento e a trabalhar em equipe. A Figura 1 demonstra como se dá essa articulação. 


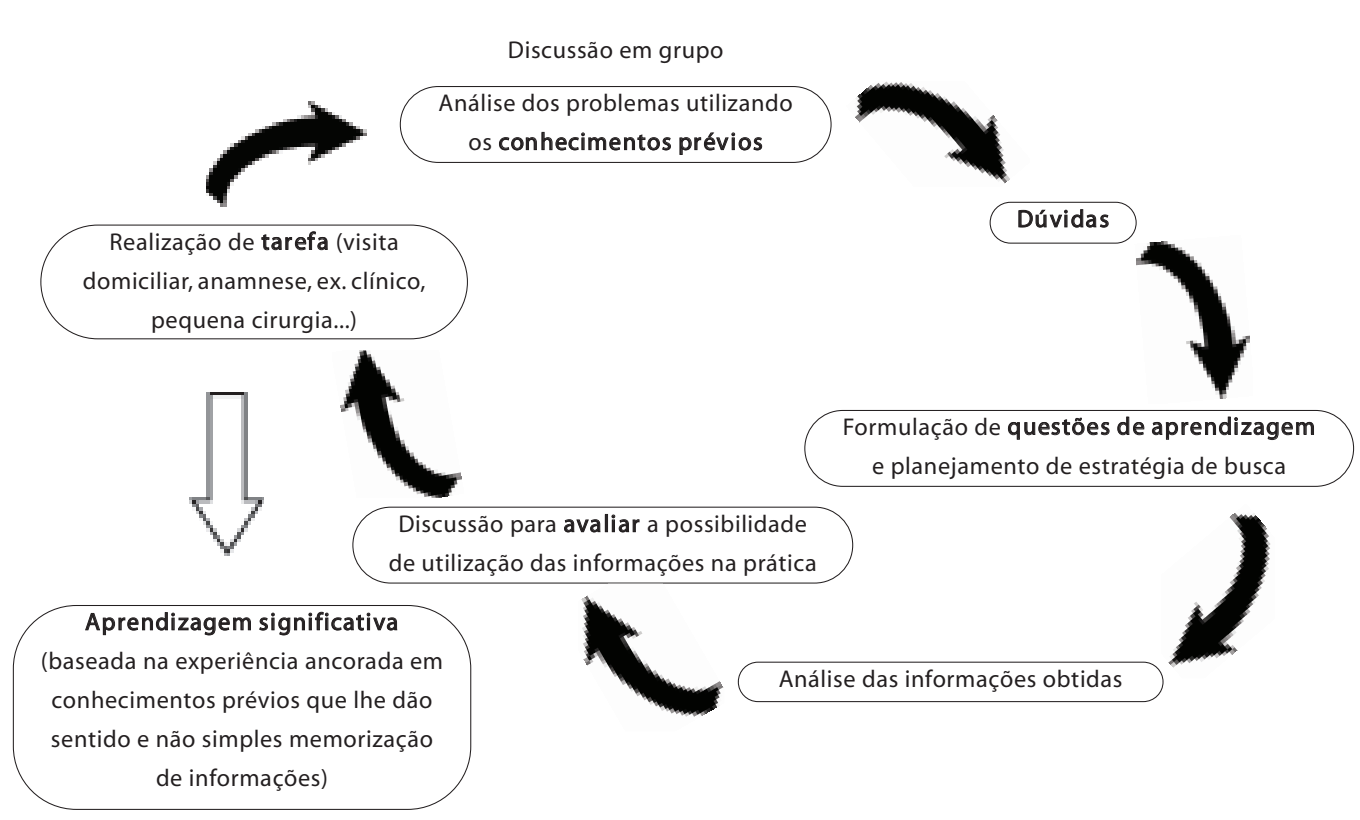

A construção do conhecimento pela participação ativa do estudante se configura, assim, por meio da realização de todas as etapas preconizadas pelo método evidenciando a construção da ação/reflexão/ação, corroborando o projeto pedagógico e o currículo do curso orientado pela competência profissional que prevê a vivência da prática, a teorização e a atuação na prática.

Ao vivenciar o cenário de prática, tendo a assistência farmacêutica como campo de atuação, os estudantes realizam tarefas de acordo com a complexidade e autonomia em que se encontram. Essa etapa do processo é de fundamental importância, pois servirá como ponto inicial da realização do processo pedagógico.

Após a realização das tarefas e da vivência dos processos de trabalho, os estudantes se encontram em grupo e na presença do professor relatam suas vivências, suas dificuldades e suas vitórias diárias (confronto experiencial). Nessa etapa, ele aprende a conviver com o desafio do que acha que sabe, do que sabe e do que não sabe.

Depois dos relatos de todos os estudantes, é realizada uma reflexão sobre as necessidades de saúde percebidas em cada caso. Essas reflexões suscitam questões como: de que maneira foram conduzidos os casos? O que poderíamos ter feito de diferente? E, principalmente, como nos sentimos em relação àquela situação (síntese provisória)? 
Com base nessas reflexões, são elaboradas perguntas que buscam uma integração dos conhecimentos biopsicossociais e das necessidades de saúde da população e servem de norte para a pesquisa (busca de informações).

Após uma semana, em um segundo encontro, cada estudante apresenta o que estudou sobre o assunto, como respondeu às perguntas, que novidades percebeu, que novas formas de lidar com as descobertas e com o sentimento por ter 'crescido' em relação ao assunto e à forma de construir o conhecimento (nova síntese). São discutidas, então, as estratégias de intervenção e encaminhadas à equipe de saúde (aplicação à realidade).

A avaliação é feita em todos os momentos do estágio: no atendimento ao paciente, na visita domiciliar, na ação educativa, durante o desenrolar do ciclo (avaliação), na apresentação das experiências, na apresentação da pesquisa, enfim, é um somatório de cada atuação, sempre procurando valorizar o desenvolvimento do estudante.

Acredita-se, assim, que a problematização como estratégia pedagógica possibilita o processo de ensino/aprendizagem, centrado nos estudantes, tornando-os mais ativos, independentes, criativos, pensadores críticos, cooperativos, capazes de avaliarem os seus progressos, com desenvolvimento de boa capacidade de comunicação, bom relacionamento interpessoal, hábitos e técnicas para continuar aprendendo ao longo da vida de maneira interdisciplinar, abordando todos os níveis de atenção à saúde com competência profissional.

Ao realizar esse processo, o estudante entra em contato com um modelo que lhe possibilita, no exercício profissional, interagir melhor com o paciente na construção de uma aliança terapêutica (Marinker, 1997; Weiss e Britten, 2003), pois considera o outro como sujeito integral nas dimensões biopsicossociais. Essa abordagem está alicerçada em um novo conceito de aquisição de conhecimento, em que o papel do farmacêutico é o de apoiar o paciente na construção do seu próprio conhecimento e das atitudes, visando ao uso dos seus medicamentos (Raynor et al., 2001; Kansanaho et al., 2004, 2005).

O objetivo deste trabalho4 é realizar uma reflexão crítica da formação de assistência farmacêutica no cenário de atenção primária à saúde, tendo o método ativo de ensino-aprendizagem da problematização como estratégia pedagógica no desenvolvimento de competências profissionais.

\section{Método}

Trata-se de uma análise de pesquisa avaliativa, o que, segundo Contandriopoulos et al. (1997, p. 37), significa “examinar através de um procedimento científico as relações que existem entre os diferentes componentes de uma intervenção" e, para tanto, se utilizarem as abordagens de pesquisa 
qualitativa que pretendem se aproximar de uma realidade com a finalidade de conhecê-la (Minayo e Sanches, 1993).

O campo é o recorte espacial que corresponde à abrangência, em termos empíricos, ao objeto de estudo; compreende o contexto no qual se desenvolve o objeto, sendo o espaço onde se obterão os dados empíricos sobre (Minayo, 2000, p. 105).

Assim, o campo determinado para este estudo foi aquele no qual se desenvolveram as atividades de assistência farmacêutica, no cenário de atenção primária à saúde, do curso de Farmácia da Faculdade de Ciências Médicas e da Saúde de Juiz de Fora (FCMS/JF). Para isso, foram analisadas transcrições dos instrumentos de avaliação aplicados durante o desenvolvimento do projeto de construção e acompanhamento do processo avaliativo das mudanças nas escolas da área da saúde (Lampert et al., 2009), aprovado por comitê de ética.

O método utilizado para o exame minucioso dos dados foi o da análise de conteúdo, modalidade temática, com ênfase na abordagem qualitativa de pesquisa social (Gomes et al., 2005). Esta técnica consiste em descobrir os 'núcleos de sentido' que compõem a comunicação e cuja presença, ou frequência de aparição, pode significar algo para o objetivo analítico escolhido.

\section{Resultados}

Foi realizada a leitura dos instrumentos preenchidos por estudantes em busca de uma compreensão global, na qual se privilegiou não apenas o conteúdo, mas também sua lógica. Em seguida, por meio de confrontos dos instrumentos, identificou-se o núcleo de sentido e posteriormente foram identificados os eixos que estruturaram os depoimentos, em torno dos quais se agruparam características comuns (Gomes et al., 2005). O núcleo de sentido identificado a partir dos instrumentos dos estudantes foi: "Os cenários de prática propiciam a formação dentro das perspectivas do Sistema Único de Saúde e do campo de atuação do profissional farmacêutico" (Lampert, 2009).

A partir desse núcleo de sentido foram trabalhados os seguintes eixos: os locais de prática; orientação discente; reorientação da formação e da assistência, todos em função do uso da problematização como estratégia de ensino.

Em relação aos locais de prática, o ponto forte identificado na formação foi a utilização dos cenários de atenção primária, ao permitir interação e oportunidade aos estudantes para uma vivência da realidade da assistência farmacêutica. Isso propicia uma integração da escola com o serviço. Os estudantes realizam visitas domiciliares que têm proporcionado um aprendizado que favorece o desenvolvimento dos recursos cognitivos, psicomotores e afetivos e, consequentemente, a atuação desse estudante na prevenção de 
doenças e na promoção da saúde das pessoas assistidas. Os depoimentos exemplificam melhor a referida constatação:

A gente trabalhou com a família. E quando a gente entra na casa, eles nos recebem e vão conversando (...) na visão mais prática, a gente pode ajudar, por exemplo: "Ah, como que posso armazenar esse remédio?" "Eu estou tomando esse remédio certo?" (Estudante 1, $4^{\circ}$ período de Farmácia).

(...) prepara os estudantes pra trabalhar em uma equipe multiprofissional, integrada com outros cursos, não só o curso de Farmácia isolado. E também volta o profissional para o lado humanista. A gente passa a ver o paciente não como um consumidor de medicamentos. (E-2, $6^{\circ}$ período de Farmácia).

Quanto à orientação do discente, parece haver uma necessidade por parte dos estudantes em se identificar com a presença do profissional farmacêutico em alguns cenários como o de atenção primária, o que não é possível devido à falta desse profissional no serviço, fato também amplamente discutido pelos estudantes. O ciclo pedagógico da problematização é um ponto forte reconhecido.

(...) na UBS fui perguntar quem fazia dispensação do medicamento e a resposta que eu tive foi: "Quem estiver à toa." Ou seja, quem estiver à toa vai lá, entrega o medicamento. Não é dispensação do medicamento, é apenas uma entrega de medicamento (E-3, $5^{\circ}$ período de Farmácia).

Se tenho dúvidas sobre a atuação do farmacêutico, não tenho com quem esclarecer, já que na UBS não existe um farmacêutico trabalhando. Entretanto, a realização do ciclo pedagógico com o professor e os colegas possibilita a discussão sobre o processo de trabalho incluindo a reflexão da falta deste profissional na rede (E-4, $5^{\circ}$ período de Farmácia).

Em relação à reorientação da formação e à assistência, a inserção do estudante amplia sua visão sobre a integralidade da atenção. Os discursos a seguir abordam este aspecto:

(...) uma outra fortaleza também é o olhar da integralidade. O olhar a partir do contato com outros alunos e profissionais cotidianamente. Assim, não existe um olhar focado só na farmácia. É possível ver a integralidade da atenção à saúde (E-5, $5^{\circ}$ período de Farmácia).

(...) eu acho muito interessante a gente trabalhar com vários profissionais e ver a importância de cada um para melhorar a assistência em saúde (E-6, $3^{\circ}$ período de Farmácia). 
A falta de reconhecimento do governo sobre a importância da atuação do farmacêutico no setor público revela o pequeno interesse de alguns estudantes pelo SUS e pelas tarefas desenvolvidas na atenção básica. Os relatos a seguir constatam essa afirmativa:

(...) a fragilidade que eu vejo no processo, principalmente para o aspecto farmacêutico, é o fato de que o próprio governo, as próprias entidades governamentais não estabelecem essa relação com o profissional. Eles deixam as atividades que seriam do profissional farmacêutico sendo realizadas por outros profissionais. Isso mostra um total desequilíbrio do processo. A equipe e os estudantes ficam desmotivados de estarem num serviço que não valoriza esse profissional (E-7, $3^{\circ}$ período de Farmácia).

(...) nós não estamos vendo um farmacêutico fazendo parte da equipe. Então, tenho uma preocupação com o futuro, entendeu? Será que se eu tentar seguir esse caminho eu vou ter um retorno de trabalho? Será que isso vai ser mesmo de fato regularizado? Será que o farmacêutico vai entrar no PSF? (E-8, $2^{\circ}$ período de Farmácia).

Apesar desse conhecimento que o aluno cria, em relação ao SUS vejo poucos colegas dizendo que depois de graduados irão seguir esta área (E-9, $5^{\circ}$ período de Farmácia).

\section{Discussão}

Os estudantes relataram que nos cenários de prática da atenção primária eles aprendem com a experiência, atendendo os pacientes, aplicando os conhecimentos adquiridos nos anos anteriores, procurando e incorporando novos conhecimentos, desenvolvendo as habilidades e atitudes preconizadas pelas DCN. Para que esse modelo seja efetivo, entretanto, é preciso desenvolver também a capacidade de trabalhar em equipe, promovendo o compartilhamento de informações entre os diferentes profissionais, visando ao bem-estar do paciente. Essa dificuldade foi salientada, sobretudo, pela ausência de profissionais farmacêuticos nesses cenários. Para muitas escolas, as séries iniciais do curso, em que as disciplinas são ensinadas dentro das salas de aula, são o momento principal da formação.

Assim, se considerarmos o modelo flexneriano de formação na saúde, que separa o ciclo básico do ciclo profissionalizante, faz-se necessário reconhecer que, se no básico temos um exagerado conteúdo disciplinar, no profissionalizante existe ainda muito pouca estruturação pedagógica. Importante esclarecer que estruturação pedagógica não significa fazer dos cenários de prática um momento teórico em detrimento da prática, mas possibilitar que a reflexão da prática, com a presença do farmacêutico, possa 
facilitar a estruturação e a aplicação do conhecimento cognitivo apreendido até esse momento do curso.

Ivama et al. (1997) corroboram a idéia de que os cursos da área da saúde ainda estão estruturados sob um modelo pedagógico impregnado com uma visão fragmentada, para a formação tecnicista e para a especialidade.

Na utilização de métodos tradicionais de ensino ocorre a reprodução do conhecimento, sendo a ação docente fragmentada e assentada na memorização. Esse modelo ainda é o dominante na formação dos profissionais da saúde que apresentam dificuldades em utilizar outras formas de ensinar que não a de transmitir conhecimentos (Behrens, 1999).

Para Bordenave e Pereira (1991), isso vai levar a uma série de repercussões, como, em nível individual, o hábito de se tomar notas e memorizar a passividade do aluno e a falta de atitude reflexiva, de um profundo 'respeito' e mesmo de uma dependência quanto às fontes de informação, à distância entre a teoria e a prática, à tendência ao racionalismo radical e à falta de problematização da realidade. Em nível social, poderá levar à adoção inadequada de informações científicas e tecnológicas dos países desenvolvidos, à escolha indiscriminada de modelos de pensamento elaborados em outras regiões, ao individualismo e à falta de participação e de cooperação e, consequentemente, à falta de conhecimento.

Entende-se que, nesta era da 'gestão do conhecimento', a capacidade de aprender a aprender torna-se indispensável para o sucesso profissional. Buscar alternativas que possibilitem aos estudantes momentos de reflexão crítica na prática e na reestruturação do conhecimento torna-se fundamental para a formação de bons profissionais. Cabe ressaltar que isso não deve ser feito sem um referencial teórico que sustente tal opção. Educação não se faz empiricamente, e toda ação docente deve vir acompanhada de uma intencionalidade pedagógica que possa ser explicada.

A educação não comporta mais uma bagagem escolar baseada no volume de conteúdos e nas especificidades de cada profissão, o que é pouco operacional e nem mesmo adequado. O que se acumula no começo da vida deve ser constantemente atualizado e aprofundado, considerando que estamos vivendo um período no qual as mudanças ocorrem com grande velocidade. Além disso, é essencial que os profissionais possam trabalhar colaborativamente compartilhando ações e conhecimentos. Ressalta-se também que a educação, para dar resposta ao conjunto das suas missões, deve organizarse em torno de quatro aprendizagens fundamentais, que serão ao longo da vida os quatro pilares do conhecimento: aprender a conhecer (ou aprender a aprender, adquirir os instrumentos de compreensão); aprender a fazer (para poder agir sobre o meio envolvente); aprender a viver juntos (a fim de participar e cooperar com os outros em todas as atividades humanas); e aprender a ser (via essencial que integra as três precedentes) (Delors, 2000). 
A utilização da problematização propicia também a interdisciplinaridade, que possibilita ampliar a capacidade humana para compreender a realidade e os problemas que nela se apresentam. Em se tratando do conhecimento que fundamenta as práticas dos profissionais, a interdisciplinaridade favorece a articulação do conhecimento de várias áreas com os seus saberes e os seus fazeres, de forma a dar mais sentido à teoria, a ampliar a compreensão dos problemas de saúde e, consequentemente, a melhorar a prática (Aguilarda-Silva et al., 2009), como foi relatado pelos estudantes. Isso favorece uma significativa visão sobre o paciente na sua dimensão biopsicossocial, que é necessária para promover e apoiar o convívio adequado com a doença. Assim, os futuros farmacêuticos, como profissionais da saúde, poderão ajudar as pessoas a manter sua saúde e a obter o máximo de benefícios dos seus medicamentos (Hepler e Strand, 1990; Airaksinen, 1996; Anderson, 2002).

Para realizar essas atividades, os estudantes precisam desenvolver a competência profissional, o que passa, necessariamente, pela mudança de paradigma da formação e da postura do professor. Para Alarcão (2001), ser professor hoje pressupõe um conceito de formação permanente, contínua, especializada e em ação. Esse perfil de formação inacabado traz consigo novas formas de trabalhar em equipe, de ser pró-ativo, de identificar as próprias necessidades de formação e os meios para consegui-la, de usar novas tecnologias e de assumir riscos.

A concepção moderna do professor o define essencialmente como um orientador no processo de questionamento reconstrutivo no aluno, supondo obviamente que ele detenha essa mesma competência. Nesse sentido, o que mais o identifica é a pesquisa, pois, a rigor, ensinar é uma atitude decorrente de pesquisa (Demo, 2000).

O que se observa, no entanto, é que o professor no ensino superior não foi preparado para ensinar. Em decorrência disso, a sua experiência deriva principalmente do conhecimento no campo em que atua. Ao entender o exercício docente como uma prática social, a profissionalização do professor passa a ser estratégica no repensar da educação em suas dimensões institucional e social. Deve-se compreender o docente como agente transformador, sujeito do processo de ruptura e de inovação interna e externa à instituição (Stella, 2007).

De posse de um programa pedagógico, com envolvimento docente e discente, torna-se necessário escolher os cenários de ensino aprendizagem, pois eles são essenciais no desenvolvimento dessa proposta. O grande desafio para a graduação em Farmácia é transcender a estrutura estritamente técnica. Alguns autores como Batista et al. (2005), Feuerwerker (2003) e Almeida (1999) discutem sobre algumas áreas críticas para o ensino da saúde, dentre as quais a desarticulação entre teoria e prática, saúde e doença, decorrentes de um modelo que utiliza o hospital universitário 
como centro de suas atividades práticas, com decorrente ênfase nas especialidades e nos procedimentos de atenção terciária e quaternária.

O cenário de atenção primária efetivo, um dos campos de atuação do estudante de Farmácia da FCMS/JF, foi destacado como um ponto positivo. Esta é, sem dúvida, uma das discussões mais importantes nos cursos da saúde e configura-se como uma zona de conflito. Afinal de contas, é possível formar bons profissionais farmacêuticos utilizando-se também de cenários primários de atenção? Para que essas zonas de conflito possam ser superadas, vê-se como necessária uma busca por novas atitudes perante o conhecimento, no sentido de se ultrapassar a divisão disciplinar clássica do conhecimento científico, caminhando para uma concepção de interdisciplinaridade e deslocamento para novos espaços de aprendizagem com privilégio da atenção primária, como sugerido por Stein:

(...) o nível do sistema de saúde acessível a todo problema novo e necessidade, além de cuidar de problemas crônicos e condições próprias da vida, prestando atenção ao longo do tempo, centrado na pessoa mais que na enfermidade, que oferece atenção aos transtornos mais comuns e que coordena e integra o atendimento prestado em outros serviços ou por outros profissionais (Stein, 2007).

No mesmo sentido, Feuerwerker afirma:

Há necessidade de redefinir os referenciais e relações com diferentes segmentos da sociedade no sentido da universidade construir um novo lugar social, mais relevante e comprometido com a superação das desigualdades. No campo da Saúde, é indispensável que a produção de conhecimento, formação profissional e prestação de serviços sejam tomadas como indissociáveis de uma nova prática (Feuerwerker, 2003, p, 25).

Em todas as dimensões da atenção à saúde se ressaltam o acesso, a confiabilidade do paciente no serviço e no desempenho profissional adequado. Nesse contexto, deve ser destacada a Atenção Farmacêutica, definida como “aquele componente da prática farmacêutica que permite a interação do farmacêutico com o paciente com o propósito de atender às necessidades deste, relacionadas aos medicamentos" (Brasil, 2006).

Trata-se de um novo modelo de atuação do profissional farmacêutico, visando atender às necessidades da população e, realmente, realizar a socialização da profissão farmacêutica. Orientar o usuário sobre o correto uso dos medicamentos e os riscos envolvidos e desenvolver ações educativas sobre medicamentos são ações realizadas pelo farmacêutico e poderão se dar em relação a um paciente individual - geralmente com base em uma receita específica - ou a grupos, como grupos de autoajuda, portadores de doenças específicas, turmas de escolares e associação de moradores, por exemplo. 
Nesse contexto, destaca-se mais uma vez a importância da presença de profissionais farmacêuticos na rede de atenção primária, o que, sem dúvida nenhuma, propiciaria melhor abordagem dos pacientes em suas necessidades de saúde e na utilização correta de medicamentos.

Os medicamentos ocupam um papel importante nos sistemas sanitários, pois salvam vidas e melhoram a saúde e a qualidade de vida das pessoas. Existem milhões de pacientes com doenças comuns sujeitas a problemas frequentemente relacionados com os tratamentos medicamentosos. Como exemplo: falta de acesso a um tratamento adequado ou a recursos para sua aquisição; uso de medicamentos de baixa qualidade; erros de medicação (dose errada, medicamento errado, posologia errada, duração errada, falta de orientação quanto ao tratamento); baixa adesão; efeitos tóxicos por automedicação; mau uso e/ou falta de efetividade dos tratamentos etc.

Dessa forma, destacam-se alguns grandes desafios da assistência farmacêutica nos cenários de atenção primária do SUS: proporcionar melhoria no acesso da população a medicamentos eficazes, seguros, de qualidade e voltados para atender às principais necessidades sanitárias da população; garantir as condições adequadas de aquisição, de armazenamento, de dispensação e de conservação de sua qualidade; propor meios para a redução de gastos com medicamentos; promover seu uso racional; proporcionar um atendimento humanizado e especializado aos pacientes com a presença de profissionais farmacêuticos.

Atuando no sentido de promover uma Assistência Farmacêutica de qualidade, o farmacêutico, assim, poderia proporcionar maior adesão dos pacientes aos tratamentos. Desta forma, haveria melhoria na resolutividade do próprio sistema de saúde, dos indicadores de adoecimento/morte e da qualidade de vida das pessoas.

Esse é o perfil proposto nas DCN para o profissional farmacêutico formado, buscando definitivamente o desafio de consolidar a inter-relação da área de formação de recursos humanos em saúde com os serviços de assistência à saúde: com formação generalista, humanista, crítica e reflexiva, capacitado a atuar, pautado em princípios éticos, no processo de saúdedoença, em seus diferentes níveis de atenção, com ações de promoção, prevenção, recuperação e reabilitação da saúde, na perspectiva da integralidade da assistência, com senso de responsabilidade social e compromisso com o ser humano (Almeida, 1999).

Cidadania, entretanto, como promotora da saúde integral é uma questão que se apresenta em relação à consolidação dessas práticas de saúde que compõem um modelo de saúde calcado nos princípios e diretrizes do SUS. É a problemática da força de trabalho, sendo que esta deve ser enfrentada do ponto de vista tanto das práticas como da formação de pessoal. Verifica-se, ao longo dos anos de implementação do SUS, que a formação da força de trabalho em saúde proporcionada pelo aparelho formador não é adequada às necessidades de 
saúde da população brasileira nem às necessidades dos serviços (Nogueira, 2002).

Como já destacado, a presença dos estudantes de farmácia na atenção primária ressaltou ainda mais a necessidade da reorientação na formação das equipes de saúde. Assim, a proposta de um novo modelo para a formação dos profissionais da área da saúde na perspectiva crítica, ou seja, a formação crítico-reflexiva na direção da constituição de sujeitos para a transformação social é considerada uma das demandas para que se reorganizem as práticas de saúde no sentido de um sistema ancorado nos princípios e diretrizes do SUS, em que a atenção primária à saúde e a Estratégia de Saúde da Família são fundamentais. No entanto, vários aspectos apresentam-se como motivos de uma crise na formação dos recursos humanos, gerados por determinantes externos e internos aos órgãos formadores.

Nessa perspectiva, há a necessidade de se redirecionar a formação inicial e permanente, pondo-se em questão os modelos e valores a serem construídos para atender a essa reorganização. Deve-se pautar pela ética nos processos de intervenção - como direito e respeito ao ser humano - para construção e reconstrução dos processos de trabalho e competências profissionais, integrando a formação à realidade e aos serviços da comunidade com a presença de todos os profissionais necessários na equipe.

A sociedade vive em constante movimento e tensão, com forças favoráveis e desfavoráveis a cada projeto implementado. Da mesma forma, a educação vive momentos nos quais seus projetos podem servir tanto para a reprodução dos processos na sociedade de forma acrítica como para desenvolver uma formação crítica, reflexiva, comprometida e consciente para atuar a favor da emancipação das pessoas, da igualdade social e da qualidade de vida, formando-se cidadãos no seu processo de humanização.

Essa formação, além de recolocar a discussão sobre sua intencionalidade, conta com o desafio de ter domínio de conhecimentos, habilidades e atitudes para atuar com competência, com qualidade formal e política, e ao mesmo tempo responder às carências sociais. Enfim, a inserção de estudantes de farmácia na atenção primária à saúde e a adoção de metodologias ativas como a problematização estão articuladas com o mundo do trabalho, com a resolução de problemas concretos, com a formação ético-política, além do aprender a trabalhar com as incertezas, buscando a consciência nos atos/escolhas praticados.

\section{Considerações finais}

Ao fazermos a análise dos aspectos avaliativos apontados, concluímos que a problematização como método pedagógico configura-se como uma adequada estratégia pedagógica. 
A problematização enfatiza a importância da construção do próprio conhecimento com base nos 'saberes' prévios e nas realidades vividas, os quais podem fazer despontar propriedades emergentes que despertam no estudante um novo olhar sobre si mesmo, sobre o outro e sobre a relação de cuidado e das práticas em saúde como a assistência farmacêutica.

Esse modelo proposto entende a universidade como um local de encontros, como cenário cujo objetivo principal é promover ao estudante uma educação integral, voltada à sua totalidade e visando ao seu desenvolvimento psíquico, espiritual, ético, cultural e social. No processo de ensino-aprendizagem e nas relações estabelecidas durante o trabalho na atenção primária à saúde, o estudante pode se descobrir como ser integral e como parte de uma totalidade, resgatando seus conhecimentos e aplicando-os à realidade, aprendendo, assim, de modo significativo. Isso possibilita formar profissionais mais preparados para o trabalho em equipe e a integralidade da atenção à saúde, capazes de continuar a aprender durante a vida profissional.

Algumas fragilidades apontadas demonstram, entretanto, que existem desafios a ser vencidos, e a principal refere-se à introdução do profissional farmacêutico como membro da equipe de saúde na atenção primária, na otimização do trabalho, na consolidação da pactuação entre a academia e os serviços, na melhoria e articulação com o projeto pedagógico.

\section{Notas}

1 Professor da Faculdade de Medicina de Marília (Famema), Marília, São Paulo, Brasil. Doutor em Genética e Evolução pela Universidade Federal de São Carlos (UFSCar). <rinaldo_henrique@uol.com.br>

Correspondência: Rodovia BR 040, km 796, CEP 36033-005, Salvaterra, Juiz de Fora, Minas Gerais, Brasil.

2 Coordenadora e professora do curso de Farmácia da Faculdade de Ciências Médicas e da Saúde de Juiz de Fora (FCMS/JF - Suprema), Juiz de Fora, Minas Gerais, Brasil. Mestre em Química Analítica Ambiental pela Universidade Federal de Viçosa. <farmacia@suprema.edu.br >

3 Professora da Faculdade de Ciências Médicas e da Saúde de Juiz de Fora (FCMS/JF Suprema), Juiz de Fora, Minas Gerais, Brasil. Mestre em Ciências da Saúde pela Universidade Federal do Rio de Janeiro (UFRJ).<nadd@suprema.edu.br>

4 Este artigo, cujo conteúdo foi proposto e organizado por Rinaldo Henrique Aguilar da Silva, é fruto de análise, elaboração e redação comum realizada por todos os autores. 


\section{Referências}

AGUILAR-DA-SILVA, Rinaldo Henrique et al. Abordagens pedagógicas e tendências de mudanças nas escolas médicas. Revista Brasileira de Educação Médica, Rio de Janeiro, v. 33, n. 1, supl. 1, p. 53-62, 2009.

AIRAKSINEN, Marja. Customer feedback as a tool for improving pharmacy services in Finland. Finland: Kuopio University Publications A. Pharmaceutical Sciences 25. University of Kuopio, 1996.

ALARCÃO, Isabel; TAVARES, José. Paradigmas de formação e investigação no ensino superior para o terceiro milênio. In: ALARCÃO, Isabel (Org.). Escola reflexiva e nova racionalidade. Porto Alegre: Artmed, 2001. p. 177.

ALMEIDA, Márcio José de. Educação médica e saúde: possibilidades de mudança. Rio de Janeiro: Associação Brasileira de Educação Médica; Londrina: Ed. UEL, 1999.

ANDERSON, S. The state of the world's pharmacy: a portrait of the pharmacy profession. Journal of Interprofessional Care, v. 16, p. 391-404, 2002.

BATISTA, Nildo et al. O enfoque problematizador na formação de profissionais de saúde. Revista de Saúde Pública, São Paulo, v. 39, n. 2, p. 147-161, 2005.

BEHRENS, Marilda Aparecida. A prática pedagógica e o desafio do paradigma emergente. Revista Brasileira de Estudos Pedagógicos, Brasília, DF, v. 80, n. 196, p. 383402, dez. 1999.

BOND, C. Pharmacists and the multi-disciplinary health care team. In: Taylor, K.M.G.; Harding, G.; Taylor, Francis (ed.). Pharmacy Practice, p. 249-269, London, 2001.

BORDENAVE, Juan Dias; PEREIRA, Adair Martins. Estratégias de ensino-aprendizagem. Petrópolis: Vozes, 1991.
BRASIL. Ministério da Saúde. Portaria n. ${ }^{\circ}$ 176 de 8 março de 1999. Estabelece critérios e requisitos para a qualificação dos municípios e estados ao Incentivo à Assistência Farmacêutica Básica e defines valores a serem transferidos. Disponível em: <http:// dtr2001.saude.gov.br/sas/PORTARIAS/Port99/ GM/GM-0176.html>. Acesso em: 5 abr. 2010.

Ministério da Saúde. Assistência Farmacêutica na Atenção Básica: instruções técnicas para sua organização. 2. ed. Brasília, DF, 2006. (Série A. Normas e manuais técnicos).

CONTANDRIOPOULOS, André-Pierre et al. A avaliação na área da saúde: conceitos e métodos. In: HARTZ, Zulmira Maria de Araújo (Org.). Avaliação em saúde: dos modelos conceituais à prática na análise da implantação de programas. Rio de Janeiro: Editora Fiocruz, 1997. p. 29-47.

COX, K.; STEVENSON, F.; BRITTEN, N. et al. 2002. A systematic review of communication between patients and health care professionals about medicine-taking and prescribing. London: GKT Concordance Unit, King's College London, 2002.

DELORS, Jacques (Org.). Educação: um tesouro a descobrir. Relatório para a Unesco da Comissão Internacional sobre Educação para o Século XXI. 4. ed. São Paulo: Cortez, 2000.

DEMO, Pedro. Metodologia do conhecimento científico. São Paulo: Atlas, 2000.

FEUERWERKER, Laura. Educação dos profissionais de saúde hoje: problemas, desafios, perspectivas e as propostas do Ministério da Saúde. Revista Abeno, São Paulo, v. 3, n. 1, p. 24-27, 2003.

GOMES, Romeu et al. Organização, processamento, análise e interpretação dos dados: o desafio da triangulação. In: MINAYO, Maria Cecília de S.; ASSIS, Simone G; 
SOUZA, Edinilsa R. de (Org.). Avaliação por triangulação de métodos: abordagem de programas sociais. Rio de Janeiro: Editora Fiocruz, 2005. p. 185-221.

HEPLER, C.D.; STRAND, L.M. Opportunities and responsabilities in pharmaceutical care. American Journal of Health-System Pharmacy, v. 47, p. 533-543, 1990.

IVAMA, Adriana M.; BATISTA, Cleide V. M.; SILVA, Regina M. R. Repensando os estágios. Olho Mágico, Londrina, v. 4, n. 15, nov. 1997.

KANSANAHO, Heli; CORDINA, M., PUUMALAINEN, Inka; AIRAKSINEN, Marja. Practicing pharmacists' patient counseling skills in the context of reflectivity. Pharmacy Education, v. 5, n. 1, p. 19-26, 2005.

KANSANAHO, Heli; PUUMALAINEN, Inka; VARUNKI, M.; AIRAKSINEN, Marja; ASLANI, P. Attitudes of Finnish community pharmacists toward concordance. Annals of Pharmacotherapy, v. 38, p. 1.946-1.953, 2004.

LAMPERT, Jadete Barbosa et al. Projeto de avaliação de tendências de mudanças nos cursos de graduação da área da saúde Caem/Abem. Revista Brasileira de Educação Médica, Rio de Janeiro, v. 33, supl. 1, p. 5-18, 2009.

MARINKER, M.; BLENKINSOPP, A.; BOND, C.; BRITEN, N.; FEELY, M.; GEORGE, C. et al. From compliance to concordance: achieving shared goals in medicine taking. London: Royal Pharmaceutical Society of Great Britain, 1997.

MINAYO, Maria Cecília de S. O desafio do conhecimento: pesquisa qualitativa em saúde. 7. ed. São Paulo: Hucitec, 2000, p. 105.

MINAYO, Maria Cecília de S. et al. Métodos, técnicas e relações em triangulação. In: MINAYO, Maria Cecília de S.; ASSIS, Simone G. de; SOUZA, Edinilsa R. de (Org.). Avaliação por triangulação de métodos: abordagem de programas sociais. Rio de Janeiro: Editora Fiocruz, 2005, p.71-100.

MARIN, Nelly et al. Assistência farmacêutica para gerentes municipais. Opas/OMS, 2003.
NARHI, U. Implementing the philosophy of pharmaceutical care into community pharmacy services - experiences with asthma patients in Finland (dissertation). 2001. Kuopio University Publications A. Pharmaceutical Sciences, v. 54. Disponível em: $<$ www.uku.fi/vaitokset/2001/unarhi.pdf $>$. Acesso em: 5 abr. 2010.

NOGUEIRA, Vera M. R.; MIOTO, Regina Célia T. Desafios atuais do Sistema Único de Saúde (SUS) e as exigências para os Assistentes Sociais. Serviço Social e Saúde: Formação e Trabalho Profissional, 2002. Disponível em: <www.fnepas.org.br/pdf/ servico_social_saude/texto2-4.pdf $>$. Acesso em: 5 abr. 2010.

PUUMALAINEN, Inka; KANSANAHO, Heli. Métodos de aconselhamento ao paciente, aspectos comportamentais e materiais de apoio ao aconselhamento ao paciente. In: WULIFI, Tana; AIRAKSINEN, Marja (ed.). Aconselhamento, concordância e comunicação: educação inovadora para farmacêuticos. Tradução de Carlos Cézar Flores Vidotti, Emília Vitória da Silva, Tarcísio José Palhano. Brasília, DF: Conselho Federal de Farmácia, 2009. cap. 1. p. 7-12.

RAYNOR, D. K.; THISTLETHWAITE, J. E.; HART, K.; KNAPP, P. Are health professionals ready for the new philosophy of concordance in medicine taking?. International Journal of Pharmacy Practice, v. 9, p. 81-84, 2001.

SANCHES, Odécio. Qualitativo-quantitativo: oposição ou complementaridade?. Cadernos de Saúde Pública, Rio de Janeiro, v. 9, n. 3, p. 239-248, jul./set. 1993.

STEIN, Airton Tetelbom. Práticas clínicas ressignificadas na atenção básica. In: SEMINÁRIO INTERNACIONAL DESAFIOS DO ENSINO DA ATENÇÃO BÁSICA NAS ESCOLAS DE MEDICINA. Anais... Brasília, DF: Ministério da Saúde, 18-21 jul. 2007. No prelo.

STELLA, Regina Ceres de Rosa. A prática médica no contexto das Diretrizes Curriculares Nacionais para o Curso de Medicina. São Paulo: [s.n.], 2007. 
WEISS, M.; BRITTEN, N. What is concordance?. Pharmaceutical Journal, v. 271, p. 493, 2003.

Recebido em 22/05/2010

Aprovado em 08/07/2010 